\title{
Using Value-of-Information Methods when the Disease Is Rare and the Treatment Is Expensive-The Example of Hemophilia A
}

\author{
Lusine Abrahamyan, MD MPH PhD ${ }^{7}$, Andrew R. Willan, PhD 2,3, Joseph Beyene, MSc PhD 2,3,4,5,6, \\ Marjorie Mclimont, MSC ${ }^{2}$, Victor Blanchette, MD FRCP FRCPC 2,6 , and Brian M. Feldman, MD MSC \\ FRCPC $2,3,4,7$ for the Canadian Hemophilia Primary Prophylaxis (CHPS) Study Group
}

\begin{abstract}
${ }^{1}$ Toronto Health Economics and Technology Assessment Collaborative, University of Toronto, Toronto, Ontario, Canada; ${ }^{2} \mathrm{Child} \mathrm{Health} \mathrm{Evaluative}$ Sciences Program, The SickKids Research Institute, Toronto, Ontario, Canada; ${ }^{3}$ The Dalla Lana School of Public Health, University of Toronto, Toronto, Ontario, Canada; ${ }^{4}$ Institute of Health Policy Management and Evaluation, University of Toronto, Toronto, Ontario, Canada; ${ }^{5}$ Department of Clinical Epidemiology and Biostatistics, McMaster University, Hamilton, Ontario, Canada; 'Division of Hematology/Oncology, The Hospital for Sick Children, Toronto, Ontario, Canada; ${ }^{7}$ Departments of Paediatrics, Division of Rheumatology, The Hospital for Sick Children, Toronto, Ontario, Canada.
\end{abstract}

BACKGROUND: Hemophilia A is a rare, sex-linked genetic disorder treated with intravenous administration of factor VIII (FVIII) to prevent bleeding; however, approaches vary across and within countries. Value-ofinformation (VOI) methods identify situations in which the cost-benefit evidence is sufficient to adopt one treatment strategy over another; when the evidence is insufficient, VOI methods provide the optimal sample size for additional research.

OBJECTIVE: The objective of the study was to use VOI methods in a cost-benefit decision context to evaluate the current evidence in support of using (1) alternate day prophylaxis (AP), (2) tailored prophylaxis (TP) or (3) on-demand treatment (OD) with FVIII to prevent arthropathy in children with severe hemophilia A.

METHODS: To apply VOI methods, several parameters such as incidence, time horizon for the decision, costs, and threshold values to avoid MRI-detected joint damage or arthropathy were defined. Two baseline threshold values of willingness to pay for avoiding arthropathy $-\$ 200,000$ and $\$ 400,000$-were selected for comparing the treatment strategies.

RESULTS: For threshold values $<\$ 200,000$, OD had a higher expected net benefit than either prophylaxis strategy, and the evidence was sufficient for its adoption. For threshold values $>\$ 400,0,00$ prophylaxis strategies had higher expected net benefit; however, a new trial with 38 patients per arm was needed to compare AP and TP, yielding an expected net gain of over \$17 million. In sensitivity analyses, the results were robust to assumptions regarding discount rate, trial fixed and variable costs, enrollment fraction, and the time horizon.

CONCLUSIONS: In rare diseases, evidence is often scarce and insufficient for decision making. In considering the funding of new research and patient reim-

Members listed in the online Appendix A

Electronic supplementary material The online version of this article (doi:10.1007/s11606-014-2880-3) contains supplementary material, which is available to authorized users.

Published online July 17, 2014 bursement in rare diseases, VOI methodology may provide more relevant determinations of the value and costs of additional research, compared to standard frequentist methods.

KEY WORDS: expected value of sample information; hemophilia; children; prophylaxis; costs.

J Gen Intern Med 29(Suppl 3):S767-73

DOI: $10.1007 / \mathrm{s} 11606-014-2880-3$

(C) Society of General Internal Medicine 2014

\section{INTRODUCTION}

Low patient accrual, variable disease progression, lack of objective outcomes, relatively high overall trial costs, and difficulties in regulatory approval are some of the challenges faced in conducting clinical studies of rare diseases. ${ }^{1}$ Over recent decades, regulatory bodies in several countries have implemented special policies and regulations to support research and development of drugs for rare and ultra-rare diseases. ${ }^{1}$ Although existing policies facilitate the market approval of such drugs, decisions for timely access and reimbursement are not universal and are usually made at the national, regional, state, or even provider level. ${ }^{2}$ This is because most drugs for rare diseases are expensive and do not meet currently acceptable cost-effectiveness thresholds used in resource allocation decisions. Furthermore, in some cases, even when cost-effectiveness is near acceptable threshold values, there is a great deal of parameter uncertainty, and a drug or technology may receive conditional funding with the option to revise as new evidence becomes available. ${ }^{3,4}$

Considering the difficulties in conducting research in rare diseases, future trials should be designed to maximize the difference between the expected value of the information provided by the results and the expected costs of the trial. The traditional tests of hypothesis and power considerations 
do not take this into consideration and ignore the potential costs of Type I or Type II errors. Current evidence may indicate that intervention $\mathrm{A}$ is superior to intervention $\mathrm{B}$. However, due to uncertainty in the evidence to date, the decision to adopt $\mathrm{A}$ is associated with an expected opportunity loss, and collecting additional evidence (e.g., conducting another clinical trial) is expected to reduce uncertainty.

As an alternative to the traditional approach, many authors have proposed value-of-information (VOI) methods. Grounded in decision theory, VOI methods can be used to assess the uncertainty associated with the existing evidence in the comparison of two competing interventions and identify optimal sample sizes if future research is still needed. ${ }^{5-16}$ The methods allow determination of the expected value of conducting additional research (e.g., a clinical trial) in monetary terms. The value of additional research, referred to as the expected value of sample information (EVSI), is the amount by which it reduces the expected opportunity loss associated with making decisions in the face of uncertainty. The expected total cost (ETC) of additional research is the sum of its financial and opportunity costs. The EVSI is a function of sample size, as is the cost of the trial. If for all sample sizes the EVSI is less than the cost, current evidence is sufficient for adopting $\mathrm{A}$, since it is irrational to conduct a trial where the trial cost exceeds the value. On the other hand, if for any sample size the EVSI exceeds the trial cost, current evidence is insufficient, and performing another trial is the optimal decision. Expected net gain (ENG) is the difference between $\operatorname{EVSI}(n)$ and $\operatorname{ETC}(n)$, and $n^{*}$ is the optimum sample size that maximizes this difference. More specific details regarding VOI methods are presented in online Appendix B.

The purpose of this article is to illustrate, in a decisionanalytic context, the application of VOI methods for assessing current evidence for the use of expensive health care interventions in rare diseases, in particular treatment of children with severe hemophilia A using recombinant Factor VIII (FVIII).

\section{Hemophilia and Factor VIII Treatment Strategies}

Hemophilia is a sex-linked rare genetic disorder with an annual incidence of approximately one in 5000 live male births. ${ }^{17}$ The worldwide prevalence of hemophilia is about 400,000 , and only $25 \%$ of affected patients receive adequate treatment. ${ }^{18}$ The disease is clinically manifested by repeated bleeding due to deficiency of clotting factor VIII (hemophilia A, $80-85 \%$ of cases) or factor IX (FIX) (hemophilia B). About $50 \%$ of all patients with hemophilia A have severe hemophilia, defined by FVIII levels lower than 1 or $2 \%$ and recurrent bleeds, mostly into joints - either spontaneously or after minor injuries - that over time lead to arthropathy and lifelong disability. ${ }^{19}$ The preferred treatment in many developed countries is the regular intravenous administration of FVIII to prevent bleeding; however, prophylaxis doses and regimens vary across and within countries. ${ }^{20}$ High-dose primary prophylaxis (the Malmö regimen) involves the intravenous administration of 20-40 International Units of FVIII per kilogram of body weight given on alternate days (minimum three times per week), started before the onset of any overt musculoskeletal disease. This is considered the gold standard of preventive care and is implemented in many Scandinavian and Northern European countries. ${ }^{21,22}$ Although the World Health Organization, the National Hemophilia Foundation, and the World Federation of Hemophilia recommend FVIII prophylaxis as the main approach for treating children, ${ }^{20}$ in several countries patients are treated with an on-demand regimen, that is, FVIII infusions given only when bleeding occurs.

Hemophilia $\mathrm{A}$ is a very expensive disease to treat, primarily due to the high cost of FVIII, which accounts for $90 \%$ of total treatment costs in hemophilia. ${ }^{23,24}$ In a study in France, the mean annual per-patient treatment cost with on-demand therapy was estimated to be USD $\$ 73,029 .{ }^{25}$ In Germany, where guidelines advocate alternate day prophylaxis for patients with severe hemophilia A, the average annual costs vary from $€ 40,000$ to $€ 120,000$ per patient. $^{26}$

There have been only two randomized clinical trials (RCT) of primary full-dose prophylaxis versus on-demand therapy in young boys with severe hemophilia $A .{ }^{27,28}$ The first multi-centered trial was conducted in the United States and compared alternate day prophylaxis (AP, $25 \mathrm{IU} / \mathrm{kg}$ ) with on-demand (OD) or enhanced episodic treatment among boys less than 30 months old with severe hemophilia $\mathrm{A}^{27}$ By the age of 6 years, $93 \%(25 / 27)$ of patients in the AP arm and $55 \%(16 / 29)$ in the OD arm did not have magnetic resonance imaging (MRI)-detected joint damage. Using an estimate of FVIII cost of USD \$1 per unit, the average (standard error) per-patient treatment cost was $\$ 352,793(\$ 26,597)$ for patients in the AP arm and $\$ 113,237(\$ 11,404)$ for patients in the OD arm. The second was an RCT in Italy that found that after 10 years, only $29 \%$ of children on alternate day prophylaxis with FVIII developed radiographic arthropathy compared with $74 \%$ of children randomized to episodic treatment $(p<0.05) .{ }^{28}$ Arthropathy was not assessed by MRI in this study.

Concurrent with the U.S. study, a single-arm, multicenter trial in Canada enrolled patients who met the same eligibility criteria, but were treated with tailored prophylaxis (TP). All patients in this cohort $(n=24)$ started with onceweekly prophylaxis and escalated to twice-weekly or alternate day prophylaxis if the bleeding pattern met specified escalation criteria. ${ }^{29}$ At 5 years of follow-up, $40 \%$ of subjects were on once-weekly prophylaxis, $32 \%$ 
were on twice-weekly prophylaxis, and $28 \%$ on alternateday prophylaxis. At the time of MRI joint assessment, children in the Canadian cohort were 8.8 years old (range $6.2-11.5$ years) and 12 out of 24 patients (50\%) had MRIdetected osteochondral joint damage. ${ }^{30}$

A recent systematic review of hemophilia treatment strategies by the Swedish Council on Health Technology Assessment concluded that there is insufficient evidence regarding the optimum time to start FVIII treatment, the optimum dose and dose interval, and its cost-effectiveness. ${ }^{31}$ The objective of this study was to use the information from the U.S. and Canadian studies to evaluate if it is worth to conduct another trial of FVIII prophylaxis in children with severe hemophilia A.

\section{METHODS}

The net benefit of a health care intervention is defined as the mean measure of health outcome times the threshold value of the willingness to pay for a unit of health outcome minus the mean cost. In this formulation, the measure of health outcome is scaled so that larger values are more desirable. In the hemophilia example, the measure of effectiveness is scored as 1 if a patient does not have MRI-detected joint damage/arthropathy and 0 if he does. Thus, the estimated mean measure of health outcome is simply the proportion of patients free of joint damage. The threshold value $(\lambda)$ is determined by how much a decisionmaker would be willing to pay (in the current example) to avoid one case of hemophilic arthropathy.

As described in online Appendix B, EVSI is the reduction in expected opportunity loss per patient (if a new trial is conducted) multiplied by the number of patients (N) who would benefit from the decision. Since FVIII prophylaxis is recommended for children who are less than 2 years old and have had no more than one joint bleed, the decision will affect newly diagnosed or incident cases of hemophilia. $\mathrm{N}$ directly depends on the incidence of the disease, trial duration, and time horizon for the decision. Since EVSI increases with incidence, the application of
VOI methods in a rare disease may often result in a situation where EVSI is less than ETC, leading to the conclusion that current evidence is sufficient for decision making. By contrast, standard frequentist methods are independent of incidence and may lead to unnecessary research.

\section{Applying VOI Methods in Hemophilia}

Consider the possibility of conducting a new, multi-center randomized controlled trial (RCT) in North America and enrolling patients with the same eligibility criteria as the previous trials, namely children with severe hemophilia A, less than 30 months old, with no joint damage and no clotting factor inhibitors. The combined annual incidence (k) in Canada and the U.S. is assumed to be 200 , based on the total number of live male births in Canada ${ }^{32}$ and the U.S. ${ }^{33}$ in 2010. The summary statistics for all three treatment strategies are given in Table 1 . To estimate the MRI score at the age of six 6 yearsr the Canadian cohort, we examined clinical variables that predict MRI joint damage at the actual time of MRI assessment. Using the MRI joint score linear prediction rule at the time of MRI assessment (including target joint development, defined as at least three bleeds into the same joint within three consecutive months ${ }^{34}$ and the number of bleeds per year), the predicted number of children with MRI-detected joint damage at the age of 6 years would have been five out of 24 , corresponding to $79 \%$ with no damage $(95 \% \mathrm{CI}$ : 60 to $91 \%$ ). Considering the fact that FVIII costs vary widely in the current market depending on the country, product type, and contract negotiations, we conducted base case analysis using a cost estimate of 0.50 U.S. cents/FVIII unit.

The values of the other parameters required for VOI determinations are given in Table 2 for base-case and sensitivity analyses. It was assumed that the decision would affect only new incident cases. Following the example of the U.S. trial, the proposed study would have 5 years of follow-up, with an additional year for data analysis and dissemination of the results (i.e., $\tau=6$ ). It was assumed for each comparison that if the trial were not conducted, future patients would receive the treatment strategy with the

Table 1. Summary Statistics by Treatment Strategy

\begin{tabular}{llll}
\hline \hline & Alternate day prophylaxis ${ }^{27}$ & On-demand treatment $^{27}$ & Tailored prophylaxis $^{\mathbf{2 9}}$ \\
\hline n patients & 27 & 29 & 24 \\
Proportion without joint damage $\left(\mu_{e 0}\right)$ & 0.9259 & 0.5517 & 0.7917 \\
$\mathrm{~V}\left(\mu_{e 0}\right)$ & 0.002540 & 0.008528 & 0.006872 \\
Mean cost $\left(\mu_{c 0}\right)^{*}$ & $\$ 176,397$ & $\$ 117,651$ & $(9,067)^{2}$ \\
$\mathrm{~V}\left(\mu_{c 0}\right)$ & $(13,298)^{2}$ & $(5,700)^{2}$ & -30.06 \\
$\mathrm{C}\left(\mu_{e 0}, \mu_{c 0}\right)^{\dagger}$ & -26.81 & -21.06 & \\
\hline
\end{tabular}

* Mean costs were calculated using an estimate of 50 U.S. cent per FVIII unit

† Covariance was calculated based on a point-biserial correlation coefficient of -0.04, estimated from the Tailored Prophylaxis group

$C$ covariance; $V$ variance 
Table 2. Parameter Estimates for Sensitivity Analysis

\begin{tabular}{llll}
\hline \hline Parameters & Base-case & Low & High \\
\hline Fixed financial costs $\left(C_{f}\right)^{*}$ & $\$ 1,000,000$ & $\$ 500,000$ & $\$ 2,000,000$ \\
Variable financial costs & 5,000 & 2,500 & 10,000 \\
$\left(C_{v}\right)^{*}$ & & & \\
Enrolment fraction $(f)$ & $20 \%$ & $15 \%$ & $25 \%$ \\
Time horizon $(h)$ & 20 years & 15 years & 25 years \\
Discount rate & $0 \%$ & $3 \%$ & $5 \%$ \\
FVIII unit cost & 0.50 cent & - & 0.75 cent, \\
& & & 1.00 USD \\
\hline
\end{tabular}

* Cost estimates were taken from the Canadian tailored prophylaxis study. All costs are in U.S. dollars

highest expected net benefit. It was also assumed that if a trial was conducted, patients not in the trial would receive the treatment strategy with the lowest expected net benefit, i.e., the treatment strategy with the lowest expected net benefit was assumed to be the standard. It was also assumed that while the trial is underway, no new treatment was developed and implemented (e.g., gene therapy) during the time horizon, and that no planned interim analysis or early stopping rule would be applied. For simplicity and due to low incidence, ${ }^{27,29}$ we ignored the risk of FVIII inhibitor development and its effect on ETC.

We performed three comparisons: Comparison 1 was AP vs. TP; Comparison 2, TP vs. OD; and, Comparison 3, AP vs. OD. We also conducted two base-case analyses using threshold values of $\$ 200,000$ and $\$ 400,000$. VOI methods were used to determine whether current evidence is sufficient for decision making and, if not, the optimal sizes of future clinical trials. A sensitivity analysis was conducted to examine the robustness of some of the assumptions. An MS-Excel spreadsheet provides optimal sample size calculation for base-case scenario for Comparison 1, along with the estimations of EVSI, ETC, and ENG (available at www.andywillan.com/downloads).

\section{RESULTS}

The expected net monetary benefit of each strategy was estimated for threshold values for the health outcome (MRIdetected joint damage) ranging from $\$ 0$ to $\$ 800,000$ (Fig. 1). For threshold values lower than $\$ 200,000$, OD has a higher expected net benefit than either AP or TP. The differences between strategies are less pronounced for threshold values between $\$ 200,000$ and $\$ 400,000$, but for threshold values above $\$ 400,000$, AP and TP have a higher expected net benefit than OD.

The optimal sample size (i.e., the sample size that maximizes ENG), as a function of the threshold value, is plotted in Figure 2 for all three comparisons. In Comparison 1 (TP vs. AP), for threshold values below $\$ 360,000$ (area A), there is sufficient evidence to adopt TP (and the optimal sample size is 0 ). For threshold values greater than $\$ 550,000$ (area D), there is sufficient evidence to adopt AP. Between the values of $\$ 360,000$ and $\$ 550,000$, the EVSI exceeds ETC and the optimal sample size is positive, making a new trial worthwhile. The evidence is insufficient for decision making, and a new trial is worthwhile for values between $\$ 220,000$ and $\$ 300,000$ in Comparison 2 (OD vs. TP), and between $\$ 290,000$ and \$350,000 in Comparison 3 (OD vs. AP).

In base-case analyses with a threshold value of $\$ 200,000$, OD was the optimal treatment, since there was enough evidence to choose TP over AP, OD over TP, and OD over AP. For a threshold value of $\$ 400,000$, there was sufficient evidence to choose either prophylaxis method over OD (Comparisons 2 and 3). However, there was insufficient evidence for Comparison 1, and a new trial comparing the two prophylaxis methods was the optimal decision. Using the base-case parameters, the optimal sample size was 38 patients per arm, with an ETC of $\$ 9,341,061$ (financial cost of $\$ 1,382,000$ and opportunity cost of $\$ 7,959,061$ ) and an ENG of $\$ 17,113,972$ (Fig. 3). For comparison, if we use a traditional sample size calculation to plan a study to test the difference in mean effectiveness of TP and AP, assuming a type I error rate of 0.05 and a power of 0.80 , we would need a total sample size of 210 , or 105 patients per arm.

Table 3 contains the results of the sensitivity analyses for Comparison 1 with a threshold value of $\$ 400,000$. The change from base-case parameters to both high and low trial costs made little difference in the ENG and the optimal sample size. Applying discounting rates of $3 \%$ and $5 \%$ decreased the EVSI, ETC, and ENG. Optimum ENG remained positive for all sensitivity analyses except the unit cost of FVIII, indicating the need to conduct a new trial. The change of FVIII cost from 50 cent/unit in basecase analysis to 75 cent/unit or USD $\$ 1.00$ resulted in negative ENG, indicating sufficient evidence to adopt TP, and the cost of a trial of any size would exceed the value of the information it produced.

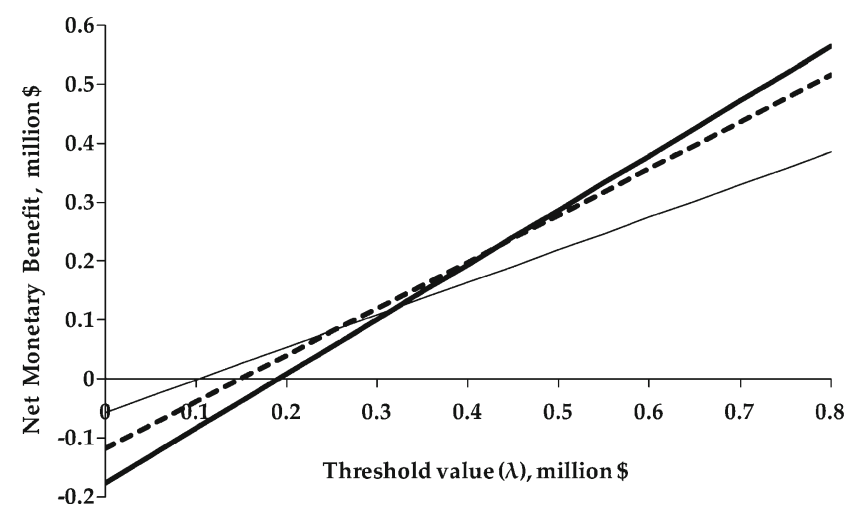

- Alternate Day Prophylaxis _ - - Tailored Prophylaxis _ On-demand therapy

Figure 1. Net monetary benefit of all treatment methods. Net monetary benefit was calculated as $\lambda * \mathbf{E}-\mathbf{C}$ where $\lambda=$ threshold value for a unit of effectiveness, $\mathrm{E}=$ effectiveness (MRI-detected joint damage) and $C=$ costs. 

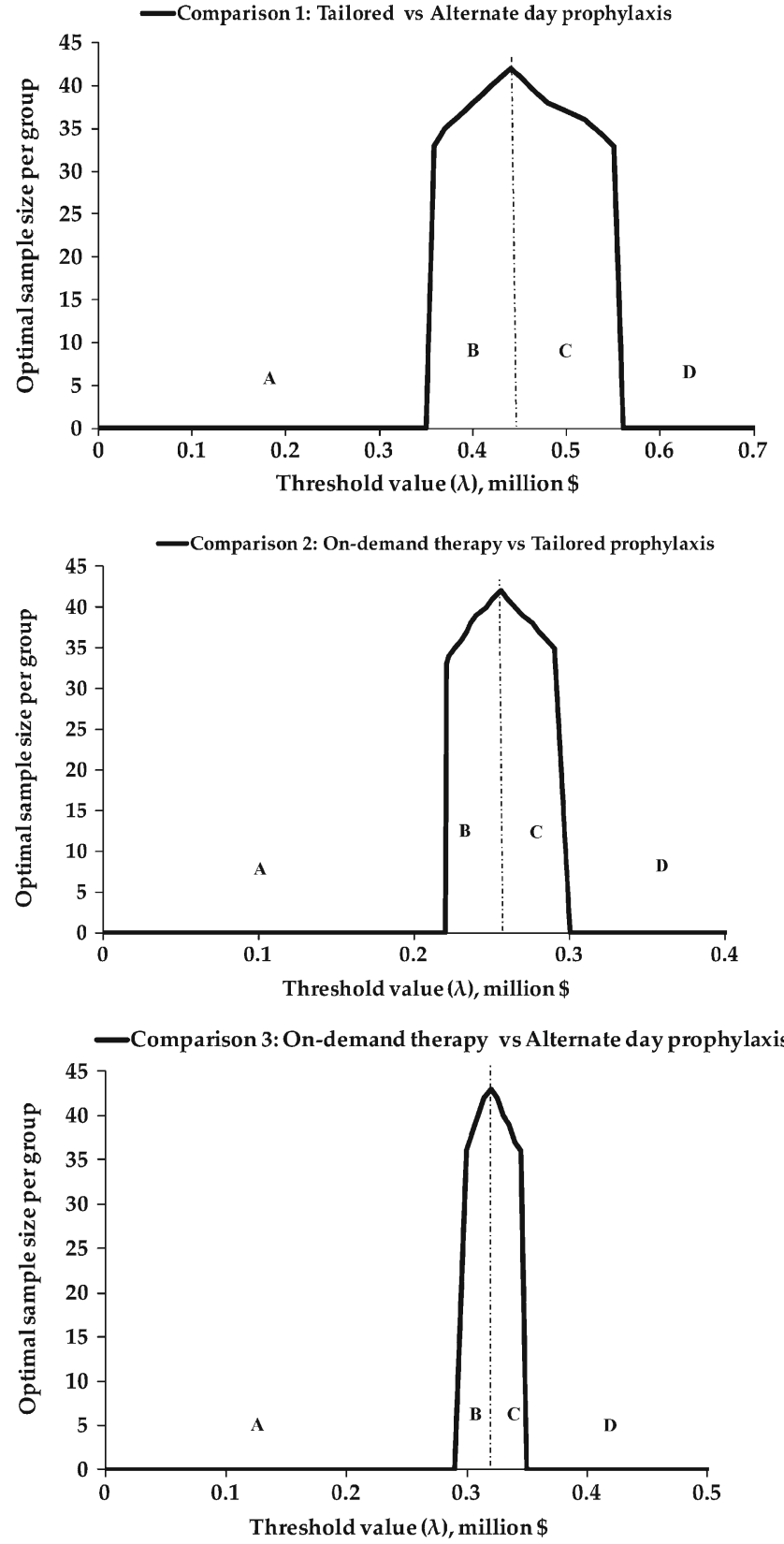

Figure 2. Optimal sample size as a function of threshold value $(\lambda)$. For all sample size and threshold value combinations, in area $A$, the optimal decision is to retain the standard treatment; in area $B$, the current evidence supports the standard treatment (lower cost, lower effectiveness), but a new trial offers a reduction of uncertainty and an expected net gain; in area $C$, the current evidence favors the experimental treatment (higher cost, higher effectiveness), but a new trial is warranted to reduce the uncertainty; and in area $D$ there is enough evidence to adopt the superior experimental treatment.

\section{DISCUSSION}

In decision analysis, the recommendation for the adoption of new health interventions is made on the basis of a positive expected incremental net benefit. However, in rare diseases, the evidence is often scarce and insufficient for decision making. In addition, although the number of

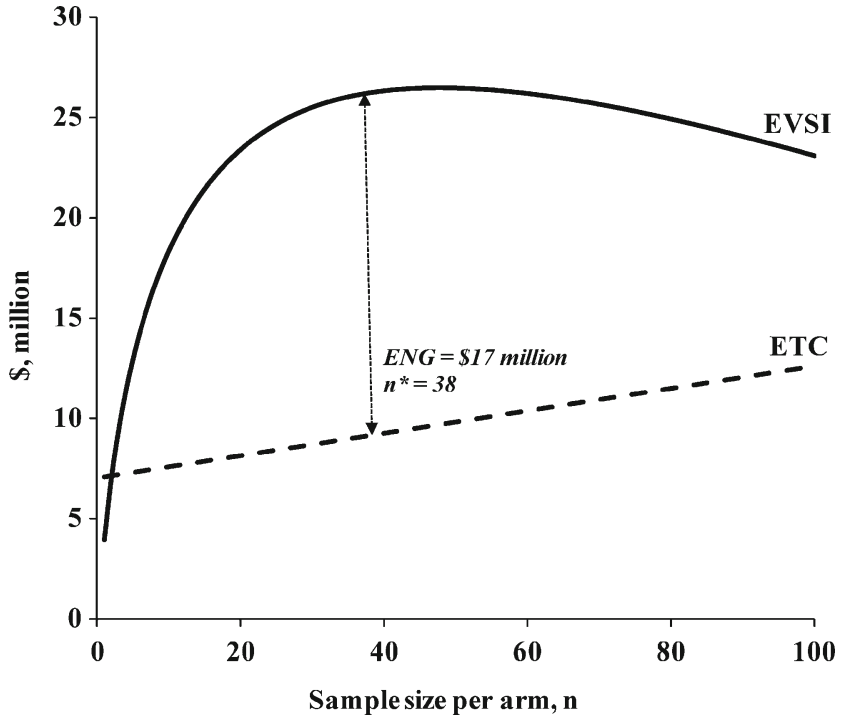

Figure 3. Expected value of sample information (EVSI) and expected total cost (ETC) as a function of sample size for Comparison 1. Comparison 1: Tailored prophylaxis vs. Alternate day prophylaxis using a threshold value of $\$ 400,000$. EVSI is the expected value of sample information, ETC is the expected total cost, $n^{*}$ is the optimal sample size, and ENG is the expected net gain.

people affected by a decision can be small, the overall opportunity loss can be significant. In considering the funding of new research and patient reimbursement in rare diseases, VOI methods may provide more relevant determinations of the value and costs of additional research than traditional sample size determinations. VOI methods take into account the current evidence, the value placed on

Table 3. Sensitivity Analyses for Comparison 1: Tailored Prophylaxis Versus Alternate Day Prophylaxis (Threshold Value $=\$ 400,000$ )

\begin{tabular}{lllll}
\hline \hline Parameters* $^{*}$ & Optimal $\boldsymbol{n}$ & $\boldsymbol{E} \boldsymbol{V S I}$ & $\boldsymbol{E T C}$ & $\boldsymbol{E N G}$ \\
\hline Base-case & 38 & $26,455,033$ & $9,341,061$ & $17,113,972$ \\
High trial costs & 37 & $26,369,240$ & $10,630,102$ & $15,739,138$ \\
Low trial costs & 37 & $26,476,818$ & $8,670,621$ & $17,806,197$ \\
$\begin{array}{c}\text { Enrollment } \\
\text { fraction: 15 \% }\end{array}$ & 31 & $24,788,219$ & $9,497,153$ & $15,291,066$ \\
$\begin{array}{c}\text { Enrollment } \\
\text { fraction: 25 \% }\end{array}$ & 44 & $27,651,540$ & $9,205,276$ & $18,446,264$ \\
$\begin{array}{c}\text { Time horizon: } \\
\text { 15 years }\end{array}$ & 28 & $15,324,493$ & $8,765,786$ & $6,558,707$ \\
$\begin{array}{c}\text { Time horizon: } \\
\text { 25 years }\end{array}$ & 47 & $38,125,228$ & $9,803,537$ & $28,321,692$ \\
$\begin{array}{c}\text { Discount rate: } \\
\text { 3\% }\end{array}$ & 38 & $21,297,611$ & $8,200,704$ & $18,027,344$ \\
$\begin{array}{c}\text { Discount rate: } \\
\text { 5\% \% }\end{array}$ & 38 & $16,965,672$ & $7,765,750$ & $9,199,922$ \\
\hline
\end{tabular}

*The optimal sample size was 0 for FVIII 75 cent and $\$ 1.00$ unit costs, indicating that current information is sufficient to favor TP over AP (i.e., no trial needed)

All costs are in U.S. dollars

${ }^{7}$ High-cost scenario assumes a fixed cost of $\$ 2,000,000$ and a variable cost of $\$ 10,000$

${ }^{*}$ Low-cost scenario assumes a fixed cost of $\$ 500,000$ and a variable cost of $\$ 2,500$

ENG expected net gain; ETC expected total cost; EVSI expected value of sample information 
health outcomes, the cost of research, the discount rate, disease incidence, and the appropriate time horizon.

Using VOI methods and the cost and effectiveness estimates of hemophilia treatment from the U.S. and Canadian trials, we compared current treatment strategies for FVIII use in hemophilia A and sought to determine whether current evidence is sufficient for making an evidence-based decision. We found that the direction of the incremental net benefit for the paired comparisons depended on the threshold value. As would be expected given the high cost of prophylactic treatment, low threshold values favored on-demand therapy, while higher threshold values favored prophylactic treatment strategies.

A recent review of FVIII treatment strategies in severe hemophilia A called for more studies to establish the optimal age to begin treatment, optimal dosage and schedule, and objective outcomes that consider also the costs of inhibitor development and cost of therapy. ${ }^{20}$ Using a specific threshold level and applying VOI methods, a decision maker can identify treatments for which there is enough evidence, treatments for which there is not enough evidence and new trials are warranted, and the optimal sample size that maximizes the ENG. For example, in our study, for a threshold level of $\$ 400,000$ to avoid arthropathy, there is sufficient evidence to choose either prophylactic strategy over on-demand therapy. However, a new trial would be needed to make an optimal choice between the two prophylaxis strategies.

The robustness of these findings can be evaluated by determining the impact of various assumptions and parameters using sensitivity analyses. We illustrate this using the example of Comparison 1 (AP vs. TP) with a threshold value of $\$ 400,000$; the results were robust for parameters of discount rate, trial fixed costs and variable costs, enrollment fraction, and the time horizon. The time horizon had a large impact on the optimal sample size, because it directly affected the number of people who would benefit from the treatment after an optimal decision was made. As expected, the unit cost of FVIII had the largest impact on the decision about treatment adoption, and an increase of the FVIII cost favors treatment modalities with the lowest FVIII utilization (i.e., TP over AP). However, the cost of FVIII varies across countries and depends - for example - on whether the product available is recombinant, plasma-derived, or virus inactivated, and whether the purchaser is able to secure a discount from the manufacturer based on the terms and duration of the contract.

Evidence shows that FVIII prophylaxis strategies are superior to on-demand therapy in preventing bleeding, further joint damage, and long-term disability in patients with severe hemophilia. ${ }^{27,35,36}$ Although even a single bleed can cause arthropathy and long-term disability, to our knowledge, no study has attempted to estimate the different decision makers' (i.e., patients, caregivers, healthcare providers, third-party payers) willingness to pay for avoiding joint damage in hemophilia. The results from past cost-utility (modeling) studies conducted in the UK, Germany, and Canada varied significantly, with incremental cost-effectiveness ratios ranging from low $£ 37,000$ per quality-adjusted life years (QALYs) to above $€ 1$ million per QALY when comparing FVIII prophylaxis with on-demand therapy. ${ }^{37}$

Our study results must be interpreted in the light of potential limitations. We made assumptions about several parameters, including patient accrual fraction, time horizon of the decision, and trial costs, that may have been incorrect. However, these assumptions were explored in sensitivity analyses, and our results were found to be robust. Recent articles about VOI methodology have tested the impact of other parameters and assumptions not considered in this study. ${ }^{5-7,15}$

Our results can be used to help plan RCTs in other rare diseases with high treatment costs. As an example, the perpatient annual cost of enzyme replacement in Gaucher disease with imiglucerase is 70,100 , with agalsidase in Fabry disease it is 109,600 , and with laronidase in mucopolysaccharidosis 1 , it is approximately $311,000 .{ }^{4} \mathrm{~A}$ careful analysis of future expected costs and benefits in planning RCTs in these diseases using VOI methods and realistic estimates of disease incidence, threshold value of health outcome, costs and time horizon might maximize both the information provided by future trials and the resulting benefits.

In conclusion, we used VOI methods to determine the expected value of sample information, the expected trial costs, and the expected net gain for therapeutic trials in boys with severe hemophilia - a rare disease with high treatment costs. We demonstrate that there remains considerable uncertainty about the optimal cost-benefit of therapy for hemophilia, and several scenarios in which future clinical trials would be the best decision. We hope that the results of the current study will help in decision making for treatment strategies in hemophilia and in planning of future studies of this condition and other rare diseases.

Conflict of Interest: The Canadian Hemophilia Primary Prophylaxis Study was funded by the Canadian Blood Services/ Héma-Quebec Partnership Fund, the MRC/Industry (Bayer) Fund, and Bayer HealthCare Pharmaceuticals, Canada.

Lusine Abrahamyan has been awarded a Canadian Arthritis Network (CAN) Graduate Student Award and Graduate Studentship from the Research Training Centre of the Hospital for Sick Children, Toronto, Canada. Brian Feldman was supported by a Canada Research Chair in Childhood Arthritis. Andrew R. Willan is funded by the Discovery Grant Program of the Natural Sciences and Engineering Research Council of Canada (grant number 44868-08). Funding sources had no role in the design, conduct, data analysis, or data interpretation of the study, nor in the decision to submit the manuscript for publication.

Prior Presentations: This work was presented as a poster at the 35th annual meeting of the Society for Medical Decision Making, 21 October 2013; and at the XXIXth International Congress of the World Federation of Hemophilia, 10 July 2010. 
Corresponding Author: Andrew R. Willan, PhD; Child Health Evaluative Sciences Program, The SickKids Research Institute, 555 University Avenue, Toronto, Ontario M5G 1X8, Canada (e-mail: andy@andywillan.com).

\section{REFERENCES}

1. Institute of Medicine. Rare disease and orphan products: Accelerating research and development. Washington, DC: The National Academies Press; 2010.

2. Drummond MF, Wilson DA, Kanavos P, Ubel P, Rovira J. Assessing the economic challenges posed by orphan drugs. Int $\mathrm{J}$ Technol Assess Health Care. 2007;23:36-42.

3. Hughes DA, Tunnage B, Yeo ST. Drugs for exceptionally rare diseases: do they deserve special status for funding? QJM. 2005;98:829-836.

4. McCabe C, Claxton K, Tsuchiya A. Orphan drugs and the NHS: should we value rarity? BMJ. 2005;331:1016-1019.

5. Eckermann S, Willan AR. Expected value of information and decision making in HTA. Health Econ. 2007; 16:195-209.

6. Eckermann S, Willan AR. Time and expected value of sample information wait for no patient. Value Health. 2008;11:522-526.

7. Willan AR, Pinto EM. The value of information and optimal clinical trial design. Stat Med. 2005;24:1791-1806.

8. Ades AE, Lu G, Claxton $\mathbf{K}$. Expected value of sample information calculations in medical decision modeling. Med Decis Making. 2004;24:207-227.

9. Claxton $\mathbf{K}$. The irrelevance of inference: a decision-making approach to the stochastic evaluation of health care technologies. J Health Econ. 1999; 18:341-364.

10. Halpern J, Brown BW Jr, Hornberger J. The sample size for a clinical trial: a Bayesian-decision theoretic approach. Stat Med. 2001;20:841-858.

11. Hornberger J, Eghtesady $\mathbf{P}$. The cost-benefit of a randomized trial to a health care organization. Control Clin Trials. 1998;19:198-211.

12. Hornberger JC, Brown BW Jr, Halpern J. Designing a cost-effective clinical trial. Stat Med. 1995;14:2249-2259.

13. Pezeshk H, Gittins J. A fully Bayesian approach to calculating sample sizes for clinical trials with binary responses. Drug Inf J. 2002;36: 143-150.

14. Welton NJ, Ades AE, Caldwell DM, Peters TJ. Research prioritization based on expected value of partial perfect information: a case-study on interventions to increase uptake of breast cancer screening. J Roy Statist Soc Ser A. 2008;171:807-841.

15. Willan AR. Clinical decision making and the expected value of informa tion. Clin Trials. 2007:4:279-285.

16. Willan AR. Optimal sample size determinations from an industry perspective based on the expected value of information. Clin Trials. 2008;5:587-594.

17. Soucie JM, Evatt B, Jackson D. Occurrence of hemophilia in the United States. The Hemophilia Surveillance System Project Investigators. Am J Hematol. 1998;59:288-294.

18. World Federation of Hemophilia (WFH). About WFH. Available at: http:// www.wfh.org/en/page.aspx?pid=642. Accessed March 6, 2014.

19. Aledort LM, Haschmeyer RH, Pettersson H. A longitudinal study of orthopaedic outcomes for severe factor-VIII-deficient haemophiliacs. The Orthopaedic Outcome Study Group. J Intern Med. 1994;236:391-399.
20. Rossbach HC. Review of antihemophilic factor injection for the routine prophylaxis of bleeding episodes and risk of joint damage in severe hemophilia A. Vasc Health Risk Manag. 2010;6:59-68.

21. Nilsson IM, Berntorp E, Lofqvist T, Pettersson H. Twenty-five years experience of prophylactic treatment in severe haemophilia A and B. J Intern Med. 1992;232:25-32.

22. Plug I, van der Bom JG, Peters M, et al. Thirty years of hemophilia treatment in the Netherlands, 1972-2001. Blood. 2004;104:3494-3500.

23. Kern M, Blanchette V, Stain AM, Einarson TR, Feldman BM. Clinical and cost implications of target joints in Canadian boys with severe hemophilia A. J Pediatr. 2004;145:628-634.

24. Smith PS, Teutsch SM, Shaffer PA, Rolka H, Evatt B. Episodic versus prophylactic infusions for hemophilia A: a cost-effectiveness analysis. J Pediatr. 1996;129:424-431.

25. Molho P, Rolland N, Lebrun T, et al. Epidemiological survey of the orthopaedic status of severe haemophilia A and B patients in France. Haemophilia. 2000;6:23-32.

26. Feldman BM, Aledort $\mathbf{L}$, Bullinger $\mathbf{M}$, et al. The economics of haemophilia prophylaxis: governmental and insurer perspectives. Proceedings of the Second International Prophylaxis Study Group (IPSG) symposium. Haemophilia. 2007;13:745-749.

27. Manco-Johnson MJ, Abshire TC, Shapiro AD, et al. Prophylaxis versus episodic treatment to prevent joint disease in boys with severe hemophilia. N Engl J Med. 2007;357:535-544.

28. Gringeri A, Lundin B, von Mackensen S, Mantovani L, Mannucci PM. A randomized clinical trial of prophylaxis in children with hemophilia A (the ESPRIT Study). J Thromb Haemost. 2011;9:700-710.

29. Feldman BM, Pai M, Rivard GE, et al. Tailored prophylaxis in severe hemophilia A: interim results from the first 5 years of the Canadian Hemophilia Primary Prophylaxis Study. J Thromb Haemost. 2006; 4: 1228-1236.

30. Kraft J, Blanchette V, Babyn P, et al. Magnetic resonance imaging and joint outcomes in boys with severe hemophilia A treated with tailored primary prophylaxis in Canada. J Thromb Haemost. 2012;10:24942502.

31. Berntorp E, Astermark J, Baghaei F, et al. Treatment of haemophilia A and $\mathrm{B}$ and von Willebrand's disease: summary and conclusions of a systematic review as part of a Swedish health-technology assessment. Haemophilia. 2012;18:158-165.

32. Statistics Canada. Table 102-4509 - Live births, by birth weight and sex, Canada, provinces and territories, annual, CANSIM (database). Available at: http://www5.statcan.gc.ca/cansim/a05?lang=eng\&id=1024509. Accessed March 06, 2014.

33. Martin JA, Hamilton BE, Ventura SJ, et al. Births: Final data for National vital statistics reports; 61(1). Hyattsville, MD: National Center for Health Statistics; 2010:2012.

34. Donadel-Claeyssens S. Current co-ordinated activities of the PEDNET (European Paediatric Network for Haemophilia Management). Haemophilia. 2006;12:124-127.

35. Fischer K, van der Bom JG, Molho P, et al. Prophylactic versus ondemand treatment strategies for severe haemophilia: a comparison of costs and long-term outcome. Haemophilia. 2002;8:745-752.

36. Khoriaty $\mathbf{R}$, Taher A, Inati A, Lee $\mathbf{C}$. A comparison between prophylaxis and on demand treatment for severe haemophilia. Clin Lab Haematol. 2005;27:320-323.

37. Farrugia A, O'Mahony B, Cassar J. Health technology assessment and haemophilia. Haemophilia. 2012;18:152-157. 